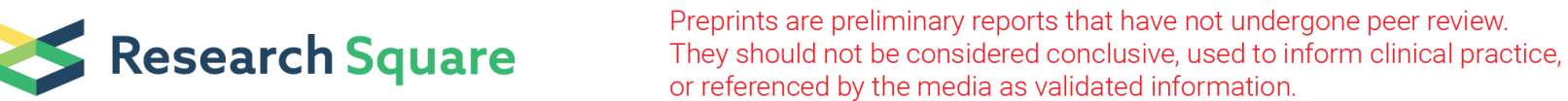

\section{Developing a routine lab test for absolute quantification of Her2 in Formalin Fixed Paraffin Embedded (FFPE) breast cancer tissues using Quantitative Dot Blot (QDB) method}

\section{Guohua Yu}

Lab of Molecular Pathology, Dept of Pathology, Affiliated Yuhuangding Hospital of Qingdao University, Yantai, Shanding

\section{Wenfeng Zhang}

Yantai Quanticision Diagnositics, Inc.,

\section{Yunyun Zhang}

Yantai Quanticision Diagnositics, Inc.,

Jiahong Lv

Yantai Quanticision Diagnositics, Inc.,

\section{Shishou Wu}

Lab of Molecular Pathology, Dept of Pathology, Affiated Yuhuangding Hospital of Qingdao University,

Yantai, Shandong

\section{Xiaolong Sui}

Lab of Molecular Pathology, Dept of Pathology, Affiated Yuhuangding Hospital of Qingdao University,

Yantai, Shandong

Jiandi Zhang ( $\sim$ jiandi.zhang@outlook.com )

Quanticision Diagnostics, Inc

\section{Fangrong Tang}

Yantai Quanticision Diagnositics, Inc.,

\section{Research}

Keywords: Biomarker, Her2, FFPE, breast cancer, QDB, quantitative, continuous, high throughput, absolute

Posted Date: January 15th, 2020

DOI: https://doi.org/10.21203/rs.2.20944/v1

License: (a) (i) This work is licensed under a Creative Commons Attribution 4.0 International License. Read Full License 


\section{Abstract}

Background: Developing immunoassay for Her2 assessment suitable for Formalin Fixed Paraffin Embedded (FFPE) samples promises improved objectivity, consistency and accuracy in daily clinical practice. Yet, this effort is hindered by lacking available technique. The feasibility of Quantitative Dot Blot (QDB) method to meet this demand was evaluated in this study.

Methods: QDB-based Immunoassay was developed for quantitative measurement of Her2 protein levels in this retrospective study using clinically validated antibody for immunohistochemistry (IHC antibody). Total protein lysates were extracted from 2X15 um FFPE slices collected from 332 breast cancer patients sequentially and non-selectively from local hospital. The absolutely quantitated Her2 levels in these samples were compared with the results from methods of Immunohistochemistry (IHC) and Fluorescence in situ hybridization (FISH) analyses using Receiver Operative Characteristics (ROC) analysis.

Results: Her2 levels measured with two IHC antibodies of EP3 and 4B5 were strongly correlated $(r=0.963$, $\mathrm{p}=0.0000)$. We also achieved area under the curve (AUC) at $0.9753 \pm 0.01026(95 \% \mathrm{Cl}: 0.9551$ to 0.9954 , $\mathrm{p}<0.0001, \mathrm{n}=322$ ) using provided results from IHC and FISH analyses, with $99.6 \%$ concordance with IHC and $88.1 \%$ with FISH at optimized cutoff of $0.261 \mathrm{nmole} / \mathrm{g}$. The concordance rate with FISH analysis was improved to $94.1 \%$ when disagreed samples were re-analyzed by third party independently.

Conclusions: We introduced the first immunoassay for absolute quantitation of protein biomarker levels in FFPE samples. This method fits well with daily clinical practice with its objectivity, simplicity and consistency, especially for local hospitals and hospitals in developing countries. The quantitatively measured Her2 protein levels in FFPE samples also allow statistically analysis at population level to meet the need of precision medicine. .

\section{Background}

Although Immunohistochemistry (IHC) is the prevailing method in biomarker assessment for solid tumors, the inherent problems with IHC, including lacking of consistency and objectivity, are also well recognized in the field[1-3]. While intensive efforts have been devoted to improve the consistency of IHC results[1,4-7], there are several ongoing efforts to develop alternative methods for absolute quantitation of biomarker protein levels objectively and consistently.

Considering the majority of clinical samples are preserved as Formalin Fixed Paraffin Embedded (FFPE) block in pathological practice, one more pre-requisite for any method suitable for routine clinical practice is that this method must be compatible with FFPE samples. In this regard, Selected Reaction Monitoring Mass Spectrometry (SRM-MS) may be the closest one to achieve this goal so far [8-10]. Nonetheless, the complicated analytical processes and high costs associated with this method remains a challenge for its usage in routine clinical practice, especially in the local clinical laboratories and clinical labs in developing countries. 
Recently, Quantitative Dot Blot (QDB) method was developed as a novel high throughput immunoassay platform for absolute quantitation of a specific protein at tissue level [11-14]. While distinctive in principle from Enzymatic linked immunosorbent assay (ELISA), QDB method nonetheless shares the same convenience, objectivity and consistency with ELISA in action. In a proof of concept (POC) study, we measured Her2 levels as absolute and continuous variables in frozen breast cancer tissues with QDB method using clinically validated antibodies for IHC (IHC antibodies) [13].

Her2 (Her2/Neu or ERBB2) is one of the most used protein biomarkers among breast cancer patients [7]. Overexpression of this protein has been found among $20 \sim 30 \%$ invasive breast patients [8]. Targeted therapies against Her2, represented by Trastuzumab (Herceptin), have found success in treating patients testing Her2 positive (Her2+), but not with those testing negative (Her2-) [9].

Currently, Her2 level is assessed through both $\mathrm{IHC}$ and Fluorescent in situ hybridization (FISH) analysis. Based on recommendations from ASCO/CAP, Her2 level is scored as $0,1+, 2+$ and $3+$, with those scoring 0 and $1+$ being defined as Her2-, and those $3+$ as Her2 + . Samples scored as $2+$ are defined as equivocal. For these samples, FISH analysis is used to further differentiate Her2 + from Her2- [10, 11].

When converted Her2 protein levels into dichotomous variables using receiver operative characteristics analysis (ROC), we found QDB results were highly consistent with those from both IHC and FISH analysis. Furthermore, results from QDB analysis eliminates the equivocal cases, as measured Her2 levels were either above or below the proposed cutoff values in our study.

The simplicity, objectivity and consistency of QDB method with frozen samples promises its potential use in daily clinical practice. This method also liberates pathologist from judging the results under the microscope. In this study, we attempted to extend QDB method to FFPE samples, as FFPE format is the last hurdle before any method can be adopted in daily clinical practice.

We measured Her2 levels in 332 FFPE samples with QDB method using EP3 and 4B5, the same two IHC antibodies used in our POC study. The consistency of results were demonstrated by comparing the results using two different IHC antibodies, EP3 and 4B5 antibodies, and comparing with those from both IHC and FISH analyses using Receiving Operative Characteristics (ROC) analysis. The correlation between Her2 protein level and several clinicopathologic parameters was also evaluated statistically.

\section{Methods}

\section{Human subjects and human cell lines}

A total of 332 Formalin Fixed Paraffin Embedded (FFPE) specimens in $2 \times 15 \mu \mathrm{m}$ slices treated for breast cancer between Jan. 2015 and Aug. 2017 were provided sequentially and non-selectively by Yuhuangding Hospital at Yantai, P. R, China. All the samples were obtained in accordance with the Declaration of Helsinki, and approved by ethics committee of Yuhuangding hospital ([2017]76 to Guohua Yu) with an 
informed consent waiver due to the use of archival tissues with retrospective clinical data which were fully anonymized before we accessed them.

MCF-7 and BT474 cell lines were purchased from the Cell Bank of Chinese Academy of Sciences (Shanghai, China), and maintained according to the provider's instruction.

\section{General reagents}

All of the chemicals were purchased from Sinopharm Chemicals (Beijing, P. R. China). Recombinant human HER2/ErbB2 protein was purchased from Sino Biological Inc. (Beijing, China). Ventana antiHER2/neu (4B5) rabbit monoclonal primary antibody was purchased from Roche Diagnostics GmbH. Rabbit anti-HER2 antibody (clone EP3) was purchased from ZSGB-BIO (Beijing, China). HRP labeled Donkey Anti-Rabbit IgG secondary antibody was purchased from Jackson Immunoresearch lab (West Grove, PA, USA). BCA protein quantification kit was purchased from Thermo Fisher Scientific Inc (Calsband, CA, USA). QDB plate was provided by Quanticision Diagnostics, Inc (RTP, USA).

\section{Preparation of FFPE tissue and cell lysates}

Two FFPE tissue slices at $15 \mu \mathrm{m}$ each $(2 \times 15 \mu \mathrm{m})$ were put into $1.5 \mathrm{ml}$ Eppendorf tubes, and deparaffinized before they were solubilized using lysis buffer (50 mM HEPES, $137 \mathrm{mM} \mathrm{NaCl}, 5 \mathrm{mM}$ EDTA, $1 \mathrm{mM} \mathrm{MgCl} 2,10 \mathrm{mM} \mathrm{Na}{ }_{2} \mathrm{P}_{2} \mathrm{O}_{7}, 1 \%$ TritonX-100, 10\% glycerol) with protease inhibitors ( $2 \mu \mathrm{g} / \mathrm{ml}$ Leupeptin, $2 \mu \mathrm{g} / \mathrm{ml}$ Aprotinin, $1 \mu \mathrm{g} / \mathrm{ml}$ pepstatin, $2 \mathrm{mM}$ PMSF, $2 \mathrm{mM} \mathrm{NaF}$ ). MCF-7 and BT474 cells were also lysed in the same lysis buffer. The supernatants were collected after centrifugation and the total amount of proteins was determined using BCA protein assay kit.

\section{QDB analysis}

The QDB process was described elsewhere with minor modifications[11, 12]. In brief, the final concentration of the FFPE tissue lysates was adjusted to $0.25 \mu \mathrm{g} / \mu \mathrm{l}$, and $2 \mu \mathrm{l} / \mathrm{unit}$ was used for QDB analysis in triplicate. The loaded QDB plate was blocked in blocking buffer ( $\%$ non-fat milk in TBST) for an hour before it was inserted into a 96-well microplate filled with $100 \mu \mathrm{l} /$ well primary antibody (for clone EP3, 1:1500 in blocking buffer; for clone 4B5, 1:10 in PBS), and incubated overnight at $4{ }^{\circ} \mathrm{C}$. Afterward, the plate was rinsed twice with TBST and washed $2 \times 10$ mins before it was incubated with a donkey antirabbit secondary antibody for 4 hours at RT. The plate was rinsed twice with TBST, washed $4 \times 10$ mins and then was inserted into a white 96 -well white plate pre-filled with $100 \mu \mathrm{l} /$ well ECL working solution for $3 \mathrm{~min}$. The chemiluminescence signals of the combined plate were quantified using Tecan Infiniti 200pro Microplate reader with the option "plate with cover".

BT474 and MCF-7 cell lysates with pre-documented Her2 level were included in all the experiments to ensure the consistency of the results. The results were accepted only when measured Her2 levels were within $10 \%$ of pre-determined levels. Samples with chemiluminescence reading less than 2 fold over blank were defined as non-detectable, entering 0 for data analysis. For those samples with the chemiluminescence reading less than that of $30 \mathrm{pg}$ Her2 recombinant protein, the narrow range ( 0 $125 \mathrm{pg}$ ) linear regression formula was used to calculate low her2 level. 


\section{FISH analysis}

Total of 16 samples were submitted to ZSGB-Bio, Inc (www.zsbio.com) at Beijing, China for FISH analysis. The detailed reports are available upon request.

\section{Statistical analysis}

All the data were presented as Mean \pm SEM. The difference between individual groups was calculated using unpaired two-tailed Student's $t$ test. $P$ value $<0.05$ was considered statistically significant. The correlation analysis was performed using either Pearson's correlation coefficient analysis or Spearman's rank correlation analysis as indicated in the figure. The performances of QDB method against IHC or FISH were evaluated using receiver operating characteristic $(\mathrm{ROC})$ analysis. All statistics were performed using GraphPad Prism software version 7.0 (GraphPad Software Inc., USA).

\section{Results}

\section{Assay development and measurement of Her2 levels as absolute and continuous variables in 332 FFPE samples}

We first developed QDB-based immunoassay for Her2 measurement in FFPE samples by defining the linear range of the assay. Total tissue lysate was extracted from $2 \times 15 \mu \mathrm{m}$ FFPE specimen by deparaffinization and solubilization with Triton-X 100 lysis buffer. Lysates from four Her $2+$ samples based on $\mathrm{IHC}$ analysis were pooled together in equal amount. The linear range of the assay was explored using pooled lysates and recombinant Her2 protein in serial dilutions in QDB measurement with both 4B5 and EP3 antibodies respectively (Additional file 1).

The Her2 protein levels were measured using total tissue lysates extracted from 332 FFPE samples with both 4B5 and EP3 antibodies within their defined linear range respectively. Samples were provided as $2 \times$ $15 \mu \mathrm{m}$ FFPE slices sequentially and non-selectively by local hospital with clinicopathological characteristics listed in Table 1. The flow diagram was shown in Fig. 1. Her2 levels measured with 4B5 and EP3 antibodies were high correlated, with $r=0.963, p=0.0000, n=332$ when evaluated with Pearson's correlation coefficient analysis (Fig. 2). For simplicity, we limited our analysis in this study with Her2 levels measured with EP3 antibody. 
Table 1

The clinicopathological characteristics of the patients.

\begin{tabular}{|c|c|c|c|c|}
\hline Variable & & No. of patients & Average \pm SEM & Percentage \\
\hline \multirow[t]{2}{*}{ Age (y) } & Total & 332 & $53.3 \pm 0.6$ & \\
\hline & $\begin{array}{l}<50 \\
\geq 50 \\
\text { Unknown }\end{array}$ & $\begin{array}{l}122 \\
209 \\
1\end{array}$ & & $\begin{array}{l}36.7 \% \\
63.0 \% \\
0.35\end{array}$ \\
\hline Histological Grade & $\begin{array}{l}\text { I } \\
\text { II } \\
\text { III } \\
\text { Unknown }\end{array}$ & $\begin{array}{l}36 \\
145 \\
119 \\
32\end{array}$ & & $\begin{array}{l}10.8 \% \\
43.7 \% \\
35.8 \% \\
9.6 \%\end{array}$ \\
\hline \multirow[t]{2}{*}{ Tumor Size (mm) } & Total & 332 & $2.3 \pm 0.6$ & \\
\hline & $\begin{array}{l}\leq 20 \\
20 \sim 50 \\
>50 \\
\text { Unknown }\end{array}$ & $\begin{array}{l}173 \\
151 \\
5 \\
3\end{array}$ & & $\begin{array}{l}52.1 \% \\
45.5 \% \\
1.5 \% \\
0.9 \%\end{array}$ \\
\hline Histological Type & $\begin{array}{l}\text { Ductal } \\
\text { Lobular } \\
\text { Other } \\
\text { Unknown }\end{array}$ & $\begin{array}{l}298 \\
9 \\
23 \\
2\end{array}$ & & $\begin{array}{l}89.85 \\
2.7 \% \\
6.9 \% \\
0.6 \%\end{array}$ \\
\hline Nodal Status & $\begin{array}{l}\text { Negative } \\
\text { Positive }\end{array}$ & $\begin{array}{l}220 \\
112\end{array}$ & & $\begin{array}{l}66.3 \% \\
33.7 \%\end{array}$ \\
\hline Her2 (IHC) & $\begin{array}{l}0 \\
1+ \\
2+ \\
3+\end{array}$ & $\begin{array}{l}77 \\
65 \\
108 \\
82\end{array}$ & & $\begin{array}{l}23.2 \% \\
19.6 \% \\
32.5 \% \\
24.7 \%\end{array}$ \\
\hline Her2 (FISH) & $\begin{array}{l}\text { Negative } \\
\text { Equivocal } \\
\text { Positive } \\
\text { Unknown }\end{array}$ & $\begin{array}{l}95 \\
6 \\
43 \\
188\end{array}$ & & $\begin{array}{l}28.6 \% \\
1.8 \% \\
13.0 \% \\
56.6 \%\end{array}$ \\
\hline
\end{tabular}

A total of 332 breast cancer FFPE tissues in $2 \times 15 \mu \mathrm{m}$ slices were provided by a local hospital. MCF-7 and BT474 cell lysates were used as internal controls. FFPE tissue lysates (about $0.5 \mu \mathrm{g} / \mathrm{unit}$ ) and cell lysates (about $0.3 \mu \mathrm{g} / \mathrm{unit}$ ) were applied onto the QDB plates at $2 \mu \mathrm{l} / \mathrm{unit}$ in triplicate for the QDB measurements with clone EP3 and 4B5 respectively. A set of serially diluted Her2 recombinant protein were included in each plate to develop plate-specific standard curve. All results were averaged from three independent experiments, with each sample in triplicate. The correlation of Her2 levels measured with 4B5 and EP3 was analyzed with Pearson's correlation coefficient analysis using Graphpad software, $r=$ $0.963, p<0.0001$.

The distributions of Her2 levels among these samples were shown in Fig. 3A. The absolute Her2 level was distributed from non-detectable (signal below two-fold of background, and enter 0 as final result) to 
as much as $31.31 \mathrm{nmole} / \mathrm{g}$. The group average was $1.953 \pm 0.254 \mathrm{nmole} / \mathrm{g}$ with 25 th and $75 \mathrm{th}$ percentile at 0 and $0.987 \mathrm{nmole} / \mathrm{g}$ respectively.

Her2 levels in all 332 breast cancer FFPE sample lysates were measured with QDB method using EP3 antibody. The lysates were diluted to about $0.25 \mu \mathrm{g} / \mu \mathrm{l}$, and then $2 \mu \mathrm{l}$ lysate was used for each sample. (A) the distribution of Her2 levels among 332 samples. Her2 levels were ranging from 0 (chemiluminescence readings less than two times the background) to $31.31 \mathrm{nmole} / \mathrm{g}$, with the mean at $1.953 \pm 0.254 \mathrm{nmole} / \mathrm{g}$. The 25th percentile was at $0 \mathrm{nmole} / \mathrm{g}$ and the 75 th percentile was at $0.987 \mathrm{nmole} / \mathrm{g}$. (B) All samples were grouped by their IHC scores provided by local hospital. The distributions of Her2 levels in each IHC group were recorded as following: 0, $0 \sim 0.205 \mathrm{nmole} / \mathrm{g}$, mean $=0.045 \pm 0.006 \mathrm{nmole} / \mathrm{g}, \mathrm{n}=77 ; 1+, 0 \sim 0.41$ $\mathrm{nmole} / \mathrm{g}$, mean $=0.049 \pm 0.008 \mathrm{nmole} / \mathrm{g}, \mathrm{n}=65 ; 2+, 0 \sim 7.25 \mathrm{nmole} / \mathrm{g}$, mean $=0.537 \pm 0.122 \mathrm{nmole} / \mathrm{g}, \mathrm{n}=$ 108; and $3+, 0.329 \sim 31.31 \mathrm{nmole} / \mathrm{g}$, mean $=7.12 \pm 0.773 \mathrm{nmole} / \mathrm{g}, \mathrm{n}=82$. The intra- and inter-CV were $8.98 \%$ and $9.89 \%$ respectively.

The samples were grouped into $0,1+, 2+$ and $3+$ groups based on $\mathrm{IHC}$ scores with average at $0.045 \pm$ $0.006(n=77), 0.049 \pm 0.008(n=65), 0.537 \pm 0.122(n=108)$, and $7.12 \pm 0.773(n=82) n m o l e / g$ respectively (Fig. 3B). The differences between each individual group were with statistical significance with one exception when analyzed using unpaired two-tailed Student's t-test $(p<0.005)$. There was no statistical difference between group 0 and group $1+$.

\section{Validation of QDB method}

The only method for absolute quantification of Her2 levels was SRM-MS, which was still in developmental stage $[8,10,15]$. Consequently, we had to rely on the results from both IHC and FISH analyses to validate our results indirectly, as the results from QDB measurement were absolute and continuous variables, while those from FISH and IHC analyses were relative and discrete variables. Therefore, receiver operating characteristic (ROC) analysis was used to evaluate QDB method with provided Her2 results from local hospital.

The samples were grouped into Her2 + and Her2- based on both IHC and FISH analyses (IHC/FISH), eliminating all the equivocal cases in the process. We achieved area under the ROC curve (AUC) at $0.9753 \pm 0.01026,95 \% \mathrm{Cl}$ at 0.9551 to 0.9954 , with $\mathrm{p}<0.0001(\mathrm{n}=322)$ (Fig. $4 \mathrm{~A})$. We also identified the optimized cutoff at $0.261 \mathrm{nmole} / \mathrm{g}$ to achieve sensitivity at $94.23 \%$ (95\% Cl: $87.87-97.85 \%)$ and specificity at $94.5 \%$ (95\% Cl: $90.58-97.12 \%)$ respectively. Using this cutoff, we achieved concordance rate with $\mathrm{IHC}$ at $99.6 \%$, and with FISH analysis at $88.6 \%(\mathrm{~K}=0.732, \mathrm{n}=140$ with Cohen's kappa analysis $)$ respectively.

Samples were separated into negative (her2-) and positive (Her2+) groups using provided results from local hospital based on the recommendations from ASCO/CAP. (A)The negative group consisted of 142 samples of $\mathrm{IHC} 0$ and $1+$ and 76 samples of IHC $2+$ with FISH negative. The positive group consisted of 82 samples of $\mathrm{IHC} 3+$ and 22 samples of $\mathrm{IHC} 2+$ with FISH positive. Samples scored as $\mathrm{IHC} 2+$ without FISH results $(n=7)$ and with FISH results equivocal $(n=3)$ were excluded. Absolute Her2 levels from QDB 
analysis were used for ROC analysis with Graphpad Prism7.0 software. The ROC curve of QDB analysis was obtained with area under the Curve (AUC) at $0.9753 \pm 0.01026 ; 95 \% \mathrm{Cl}: 0.9551 \sim 0.9954 ; \mathrm{P}<0.0001$.

(B) Samples were grouped based on their IHC scores alone, with 142 samples in the negative group (IHC 0 and $1+)$, and 82 samples in the positive group (IHC $3+$ ). Samples with IHC score of $2+$ were excluded from the analysis. The ROC curve of QDB analysis was obtained with area under the Curve (AUC) at $0.9998 \pm 0.0002 ; 95 \% \mathrm{Cl}: 0.9994 \sim 1 ; \mathrm{P}<0.0001$. (C) Samples were grouped based on FISH results alone, with 95 samples as negative (her2-) group and 43 samples as positive (Her2+) group. Equivocal cases (n =6) were excluded in the analysis. The area under the curve (AUC) was at $0.978 \pm 0.0112$, with $95 \% \mathrm{Cl}$ at $0.9561 \sim 0.9999 ; P<0.0001$. (D) Samples were grouped by IHC scores, and the suggested cutoff values from ROC analyses in (B) at $0.267 \mathrm{nmole} / \mathrm{g}$ (solid line), in (A) (C) at $0.261 \mathrm{nmole} / \mathrm{g}$ (dashed line) were shown to demonstrate the effectiveness of these cutoff values to separate samples from Her2 + to Her2groups. Her2 levels were plotted in log scale to better demonstrate the distribution of QDB results among these samples. For those samples with undetectable Her2 level, a value of $0.001 \mathrm{nmole} / \mathrm{g}$ was arbitrarily entered to avoid omitting any sample in the log scale graph.

$\mathrm{ROC}$ analyses were also performed using provided $\mathrm{IHC}$ scores alone, eliminating all the $2+$ group, and we achieved AUC at $0.9998 \pm 0.0001,95 \% \mathrm{Cl}$ at 0.9994 to 1 , with $p<0.0001(n=224)$ (Fig. 4B). The optimized cutoff was identified at 0.267 nmole/g to achieve $100 \%$ sensitivity ( $95 \% \mathrm{Cl}$ : $95.6-100 \%$ ) and $99.3 \%$ specificity (95\% Cl: $96.14-99.98 \%$ ). Likewise, for FISH analysis, we were able to achieve AUC at $0.978 \pm$ $0.0112,95 \% \mathrm{Cl}$ at 0.9561 to 0.9999 , with $\mathrm{p}<0.0001(\mathrm{n}=138$ ) (Fig. $4 \mathrm{C}$ ). The optimized cutoff was identified at $0.261 \mathrm{nmole} / \mathrm{g}$ to achieve $93.02 \%$ sensitivity (95\% Cl: $80.94-98.54 \%$ ) and $93.68 \%$ specificity (95\% Cl: 86.76-97.65\%). These cutoffs were also shown in a log scale plot of all the samples grouped by their IHC scores (Fig. 4D).

Using the $0.261 \mathrm{nmole} / \mathrm{g}$ cutoff derived from ROC analysis of IHC/FISH results, we identified 16 samples $(11.4 \%)$ in disagreement with provided FISH results. To rule out potential misdiagnosis, these samples were submitted to a third party for independent FISH analysis (Additional file. 2). We were able to recategorized 6 samples, with 2 samples as equivocal case. The overall concordance rate between QDB and IHC/FISH was also increased from 94.4-96.6\% consequently.

The correlation between Her2 copy numbers from FISH analysis, reflected by the ratio of Her2 number over chromosome 17 number (Her2/CEP17), with Her2 protein level as continuous variables was analyzed in Additional file. 3. We found a strong correlation between DNA amplification level and Her2 protein level, with $r=0.75$ with Pearson's correlation coefficient analysis $(n=122)$.

\section{Exploration of the correlation between clinicopathologic factors and Her2 as absolute and continuous variables}

The quantitated Her2 levels in FFPE samples allows us to investigate the correlation between Her2 levels as absolute and continuous variables with other clinicopathologic factors including age, histological 
grade by Nottingham grading system, tumor size and metastasis status. Her2 levels were found to be associated significantly with histological grade based on Nottingham grading system using Spearman's rank correlation analysis $(\rho=0.195, p=0.001)$, a conclusion consistent with previous studies based on IHC analysis[16, 17]. In the same study, we found that age was negatively associated with Her2 with statistical significance based on IHC analysis $(\rho=-0.117, p<0.05)$, but not based on the absolutely quantitated Her2 levels ( $\rho=-0.084, p=0.127$ ) (Additional file. 4).

Her2 distribution by histological grade was further analyzed in Fig. 5. We observed the average of these samples by Grades at $0.791 \pm 0.555,1.554 \pm 0.330,3.271 \pm 0.535 \mathrm{nmole} / \mathrm{g}$ for Grade I, Grade II, and Grade III respectively. There were statistical significance between Grade I vs Grade III $(p<0.05)$ and grade II vs Grade III ( $p=0.005)$ using unpaired two-tailed Student's t-test. We also calculated the percentage of Her2 + in each grade with $8.3 \%$ for Grade I, $29.7 \%$ for Grade II and $47.1 \%$ for Grade III. Thus, the possibility of Her2 + for Grade III patient was 5.7 fold over that of Grade I patient.

\section{Discussion}

In this study, we measured Her2 protein levels absolutely and quantitatively in 332 FFPE samples using QDB method with two clinically validated antibodies for IHC analysis. The results are objective, consistent and in high throughput format. When converted into dichotomous variables, results from QDB method showed high concordance with clinical results from both FISH and IHC analyses provided from local hospital. It is worthy of mentioning that there were no equivocal cases in QDB measurements, as the results were either above or below the proposed cutoffs, thus significantly reduce the ambiguity associated with the current prevailing methods of FISH and IHC analyses.

To the best of our knowledge, this is the first time a protein biomarker levels were measured in FFPE samples as absolute and continuous variables in an immunoassay. The assay requires minimum amount of total protein lysates extracted from FFPE slices $(0.5 \mu \mathrm{g}$ per sample for Her2). At this rate, lysates prepared from $2 \times 15 \mu \mathrm{m}$ slices from a typical FFPE block were sufficient to analyze over 20 protein biomarkers. The method also requires minimum training to be practiced routine in clinical laboratory setting.

The inherent validation steps in the analytical process, including using cell lysates with pre-determined Her2 content as quality control, also significantly reduced the inter-laboratory variations. It is perceivable the adoption of this method would significantly improve the accuracy of Her2 testing in clinical practice, especially for local laboratories, and clinical laboratories in developing countries.

It should be emphasized that QDB is not the replacement of IHC. Rather, it complements IHC in accurate assessment of protein levels of tissue biomarkers in daily clinical practice. Clearly, IHC remains irreplaceable when addressing issues relating to the cellular localization of tissue biomarkers. Nonetheless, the semi-quantitative nature of IHC analysis makes it a mission impossible to quantify the protein levels of tissue biomarkers accurately and consistency. 
QDB method and IHC analysis were both based on antigen-antibody interaction. Ideally, results from QDB analysis should match very well with those from IHC analysis when $\mathrm{IHC}$ analysis was performed properly as dichotomous variables. However, this method is fundamentally different from FISH analysis, as one analyzed at protein level (QDB), while the other one analyzed at DNA level (FISH). It is well recognized the FISH analysis has the inherent drawback of being unable to reflect faithfully changes at protein level[18]. Indeed, in this study, even after third party verification, there were still 8 samples with their QDB results different from FISH reports. Nonetheless, considering one major usage of Her2 assessment is for antibody-based targeted therapy, we believe QDB results are more clinical relevant than those from FISH analysis.

In this study and several other studies, Her2 levels showed wide distribution even among Her2 positive samples [8, 19-21]. The highest level we measured in QDB analysis was over 100 fold over the proposed cutoff value with both antibodies. Furthermore, studies based on SRM-MS method showed that both gastric and breast cancer patients with higher level of Her2 responded better to Herceptin treatment. These results emphasized the necessity to quantify Her2 levels quantitatively among cancer patients[8, 21].

\section{Conclusions}

We developed the first immunoassay to measure Her2 levels as absolute and continuous variables in FFPE samples. The success of this study supported QDB method as a reliable platform for absolute quantitation of tissue biomarkers in FFPE specimen for routine clinical use. Considering the large number of FFPE samples available worldwide, this study may signal a new direction in clinical diagnosis, where the clinical usage of tissue biomarkers will be fully explored through database-supported mathematically analysis at population level.

\section{Abbreviations}

FFPE

Formalin Fixed Paraffin Embedded

QDB

Quantitative Dot Blot

SRM-MS

Selected Reaction Monitoring Mass Spectrometry

ROC

Receiving Operative Characteristics

ELISA

Enzymatic linked immunosorbent assay

IHC

Immunohistochemistry

FISH 
Fluorescence in situ hybridization

\section{Declarations}

Ethics approval and consent to participate:

All the samples were obtained in accordance with the Declaration of Helsinki, and approved by ethics committee of Yuhuangding hospital ([2017]76 to Guohua Yu with informed consent waiver for anonymized archival tissues with retrospective clinical data.

Consent for publication:

Not applicable.

Availability of data and materials:

The data will be available upon request by writing to Jiandi.zhang@outlook.com.

Competing interests:

WZ, FT, YZ, JL \& JZ are employees of Yantai Quanticision Diagnostics, Inc., a division of Quanticision Diagnostics, Inc., who own or has filed patent applications for QDB plate, QDB method, \& QDB application in IHC analysis.

GY, SW, XS declared no conflict of interest.

Funding:

This research was funded by Yantai Regional Innovation \& Development fund ZCQ18115 to JZ; Binzhou Medical University Fund to JZ; and Natural Science Fundation of Shandong Province ZR2015HQ013 to GY.

Authors' contributions:

FT, WZ, \& YZ performed all the experiments, collected data \& performed data analysis, GY, SW, \& XS provided clinical samples \& performed data analysis, JL participated in data collection, GY, FT \& JZ participated in data interpretation, FT supervised all the experiments, JZ designed \& supervised the overall study and drafted the manuscript.

Acknowledgements.

All the contributions have been properly acknowledged in the manuscript.

\section{References}


1. Laenkholm A-V, Grabau D, Møller Talman M-L, Balslev E, Bak Jylling AM, Tabor TP, et al. An interobserver Ki67 reproducibility study applying two different assessment methods: on behalf of the Danish Scientific Committee of Pathology, Danish breast cancer cooperative group (DBCG). Acta Oncologica. 2018;57:83-9.

2. Gown AM. Current issues in ER and HER2 testing by IHC in breast cancer. Mod Pathol. 2008;21:S815.

3. Gown AM. Diagnostic Immunohistochemistry: What Can Go Wrong and How to Prevent It. Archives of Pathology \& Laboratory Medicine. 2016;140:893-8.

4. Polley M-YC, Leung SCY, McShane LM, Gao D, Hugh JC, Mastropasqua MG, et al. An International Ki67 Reproducibility Study. J Natl Cancer Inst. 2013;105:1897-906.

5. Wolff AC, Hammond MEH, Hicks DG, Dowsett M, McShane LM, Allison KH, et al. Recommendations for Human Epidermal Growth Factor Receptor 2 Testing in Breast Cancer: American Society of Clinical Oncology/College of American Pathologists Clinical Practice Guideline Update. Journal of Clinical Oncology. 2013;31:3997-4013.

6. Wolff AC, Hammond MEH, Schwartz JN, Hagerty KL, Allred DC, Cote RJ, et al. American Society of Clinical Oncology/College of American Pathologists guideline recommendations for human epidermal growth factor receptor 2 testing in breast cancer. J Clin Oncol. 2007;25:118-45.

7. Wolff AC, Hammond MEH, Allison KH, Harvey BE, Mangu PB, Bartlett JMS, et al. Human Epidermal Growth Factor Receptor 2 Testing in Breast Cancer: American Society of Clinical Oncology/College of American Pathologists Clinical Practice Guideline Focused Update. JCO. 2018;JC0.2018.77.8738.

8. Nuciforo P, Thyparambil S, Aura C, Garrido-Castro A, Vilaro M, Peg V, et al. High HER2 protein levels correlate with increased survival in breast cancer patients treated with anti-HER2 therapy. Mol Oncol. 2016;10:138-47.

9. Hembrough T, Thyparambil S, Liao W-L, Darfler MM, Abdo J, Bengali KM, et al. Selected Reaction Monitoring (SRM) Analysis of Epidermal Growth Factor Receptor (EGFR) in Formalin Fixed Tumor Tissue. Clin Proteomics. 2012;9:5.

10. Steiner C, Tille J-C, Lamerz J, Geijtenbeek SK van, McKee TA, Venturi M, et al. Quantification of HER2 by Targeted Mass Spectrometry in Formalin-Fixed Paraffin-Embedded (FFPE) Breast Cancer Tissues. Molecular \& Cellular Proteomics. 2015;14:2786-99.

11. Tian G, Tang F, Yang C, Zhang W, Bergquist J, Wang B, et al. Quantitative dot blot analysis (QDB), a versatile high throughput immunoblot method. Oncotarget. 2017;8:58553-62.

12. Qi X, Zhang Y, Zhang Y, Ni T, Zhang W, Yang C, et al. High Throughput, Absolute Determination of the Content of a Selected Protein at Tissue Levels Using Quantitative Dot Blot Analysis (QDB). JoVE (Journal of Visualized Experiments). 2018;e56885-e56885.

13. Zhang W, Yu G, Zhang Y, Tang F, Lv J, Tian G, et al. Quantitative Dot Blot (QDB) as a universal platform for absolute quantification of tissue biomarkers. Analytical Biochemistry. 2019;576:42-7.

14. Zhu Y, Qi X, Yu C, Yu S, Zhang C, Zhang Y, et al. Identification of prothymosin alpha (PTMA) as a biomarker for esophageal squamous cell carcinoma (ESCC) by label-free quantitative proteomics 
and Quantitative Dot Blot (QDB). Clin Proteomics [Internet]. 2019 [cited 2019 Apr 21];16. Available from: https://www.ncbi.nlm.nih.gov/pmc/articles/PMC6449931/

15. Catenacci DVT, Liao W-L, Zhao L, Whitcomb E, Henderson L, O'Day E, et al. Mass-spectrometry-based quantitation of Her2 in gastroesophageal tumor tissue: comparison to IHC and FISH. Gastric Cancer. 2016;19:1066-79.

16. Aman NA, Doukoure B, Koffi KD, Koui BS, Traore ZC, Kouyate M, et al. HER2 overexpression and correlation with other significant clinicopathologic parameters in Ivorian breast cancer women. BMC Clin Pathol. 2019;19:1.

17. Zhen H, Yang L, Li L, Yu J, Zhao L, Li Y, et al. Correlation analysis between molecular subtypes and Nottingham Prognostic Index in breast cancer. Oncotarget. 2017;8:74096-105.

18. Tse $\mathrm{CH}$, Hwang HC, Goldstein LC, Kandalaft PL, Wiley JC, Kussick SJ, et al. Determining True HER2 Gene Status in Breast Cancers With Polysomy by Using Alternative Chromosome 17 Reference Genes: Implications for Anti-HER2 Targeted Therapy. JCO. 2011;29:4168-74.

19. Cheng H, Bai Y, Sikov W, Sinclair N, Bossuyt V, Abu-Khalaf MM, et al. Quantitative measurements of HER2 and phospho-HER2 expression: correlation with pathologic response to neoadjuvant chemotherapy and trastuzumab. BMC Cancer. 2014;14:326.

20. Denkert C, Huober J, Loibl S, Prinzler J, Kronenwett R, Darb-Esfahani S, et al. HER2 and ESR1 mRNA expression levels and response to neoadjuvant trastuzumab plus chemotherapy in patients with primary breast cancer. Breast Cancer Research. 2013;15:R11.

21. An E, Ock C-Y, Kim T-Y, Lee K-H, Han S-W, Im S-A, et al. Quantitative proteomic analysis of HER2 expression in the selection of gastric cancer patients for trastuzumab treatment. Ann Oncol. 2017;28:110-5.

\section{Additional Files}

\section{Additional file. 10Defining the linear range of QDB measurements with two anti-Her2 antibodies, EP3 and 4B5.}

(A, C) Defining the linear range of QDB method for analysis of breast cancer FFPE tissue lysates. Human breast cancer FFPE tissue blocks in two $15 \mu \mathrm{m}$ slices $(2 X 15 \mu \mathrm{m})$ were obtained from a local hospital, and the tissue lysates were prepared as described in Methods. Breast cancer FFPE tissue lysates prepared from 4 samples with an IHC score of 3+ were mixed in equal amounts based on the BCA assay. The pooled lysate was serially diluted, as indicated in the figure, and supplemented with $0.5 \mu \mathrm{g} / \mu \mathrm{llgG}$-free BSA solution to ensure equal loading of the samples. The lysates were then applied onto the QDB plate at $1 \mu \mathrm{g} / \mathrm{unit}$ in triplicate for QDB analysis using two anti-Her2 antibodies, EP3 and 4B5 respectively. (B, D) Defining the linear range of QDB method for analysis of purified Her2 recombinant protein. The Her2 recombinant protein was serially diluted supplemented with $0.5 \mu \mathrm{g} / \mu \mathrm{l} \mathrm{lgG}$-free BSA solution. The diluted solution was then used for QDB analysis at $1 \mu \mathrm{g}$ /unit in triplicate for measurement with EP3 and 4B5 antibodies respectively. 
Additional file. 2: Third party analysis of samples in disagreement between QDB and FISH results provided from local hospital.

The Her2 levels measured with QDB method were used to separate samples into Her2+ and Her2- using suggested cutoff value at $0.261 \mathrm{nmole} / \mathrm{g}$. There were 16 samples identified with disagreed QDB and FISH results. These samples were sent to a third party to rule out potential misdiagnosis, and the results were shown at the right column. All the positive results, either from FISH or QDB analyses, were in bold. The concordance rate would be improved to $94.2 \%$ ( $k=0.865$ using Cohen's Kappa analysis) when the results from third party were incorporated in the analysis.

Additional file. 30Correlation between Her2 gene copy numbers (Her2/CEP17) and their protein levels.

The correlation was assessed with Pearson correlation coefficient analysis using Her2 protein levels from QDB analysis and Her2/CEP17 ratio from FISH analysis $(r=0.75, p<0.0001)$. The statistical analysis was performed using Graphpad 7.0.

Additional file. 4: Assessment of relationships between clinicopathologic features and Her2 levels by QDB and IHC analysis respectively.

Her2 levels, assessed either by QDB method as absolute and continuous variables, or IHC analysis as relative and discrete variables, were used to explore the putative association with clinicopathological features using Spearman's rank correlation analysis using Graphpad 7.0. The statistically significant associations were indicated in the figure. The histological grades based on Nottingham grading system were found to be associated with Her2 levels assessed either by IHC $(r=0.195, p<0.001)$ or QDB measurement $(r=0.204, p<0.0005)$ with statistical significance.

\section{Figures}




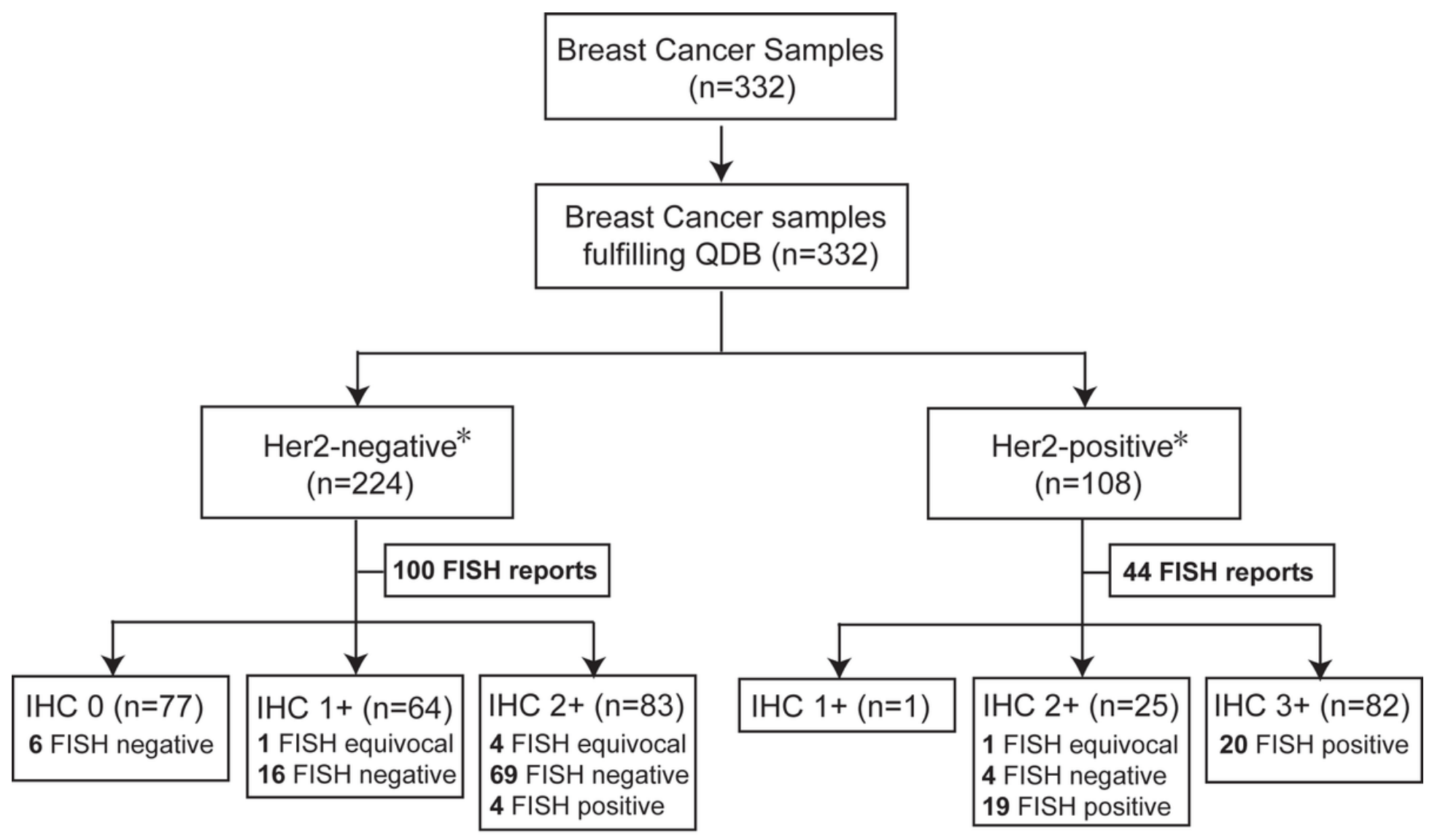

* based on cutoff value at $0.267 \mathrm{nmole} / \mathrm{g}$ derived from ROC analysis with results from IHC analysis when analyzed with EP3 antibody

\section{Figure 1}

The flow diagram of participants. 


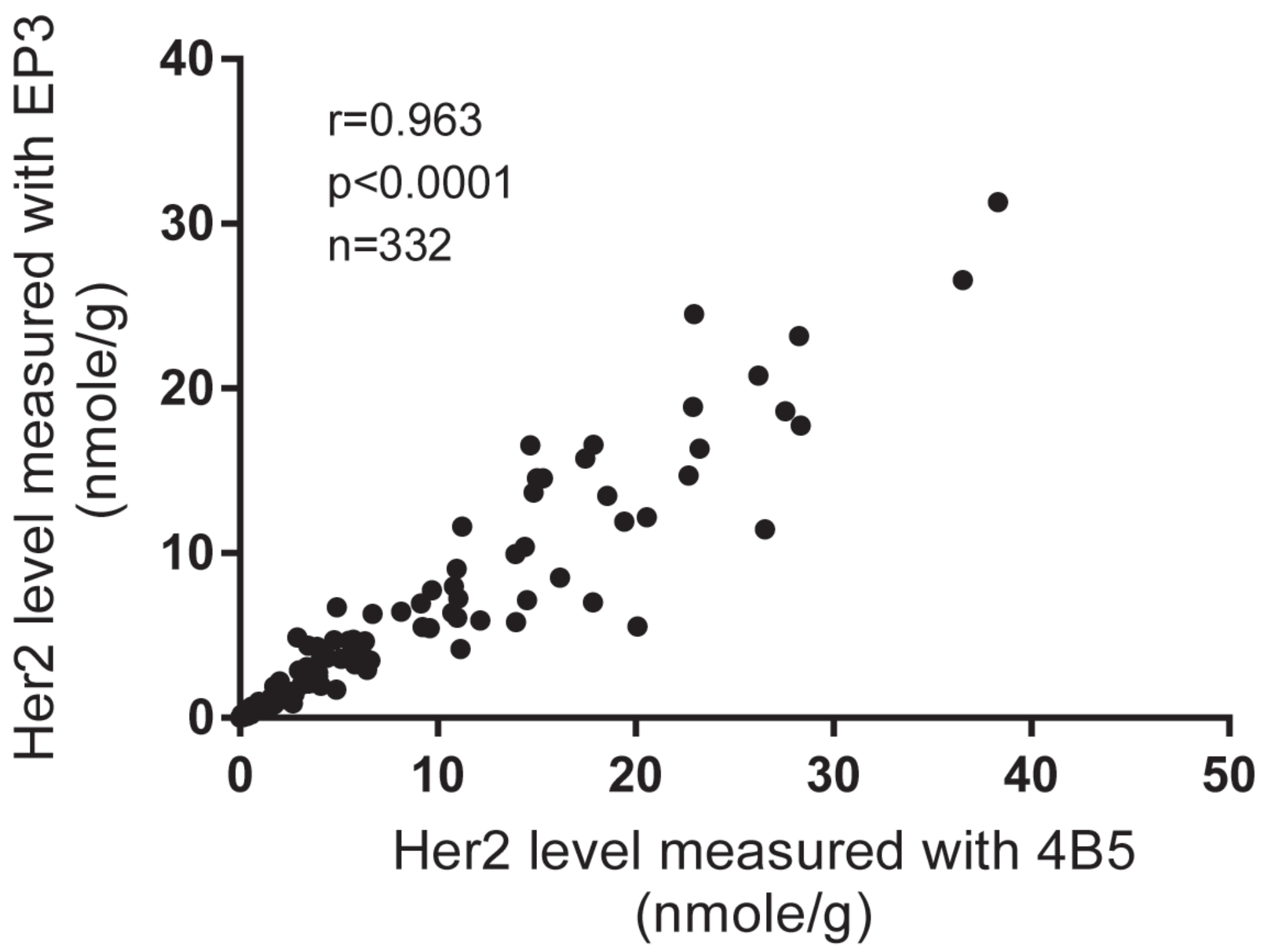

Figure 2

Correlation of Her2 levels measured with 4B5 and EP3 antibodies. A total of 332 breast cancer FFPE tissues in $2 \times 15 \mu \mathrm{m}$ slices were provided by a local hospital. MCF-7 and BT474 cell lysates were used as internal controls. FFPE tissue lysates (about $0.5 \mu \mathrm{g} \nabla / \mathrm{unit}$ ) and cell lysates (about $0.3 \mu \mathrm{g} / \mathrm{unit}$ ) were applied onto the QDB plates at $2 \mu \mathrm{l} / \mathrm{unit}$ in triplicate for the QDB measurements with clone EP3 and 4B5 respectively. A set of serially diluted Her2 recombinant protein were included in each plate to develop plate-specific standard curve. All results were averaged from three independent experiments, with each sample in triplicate. The correlation of Her2 levels measured with 4B5 and EP3 was analyzed with Pearson's correlation coefficient analysis using Graphpad software, $r=0.963, p<0.0001$. 

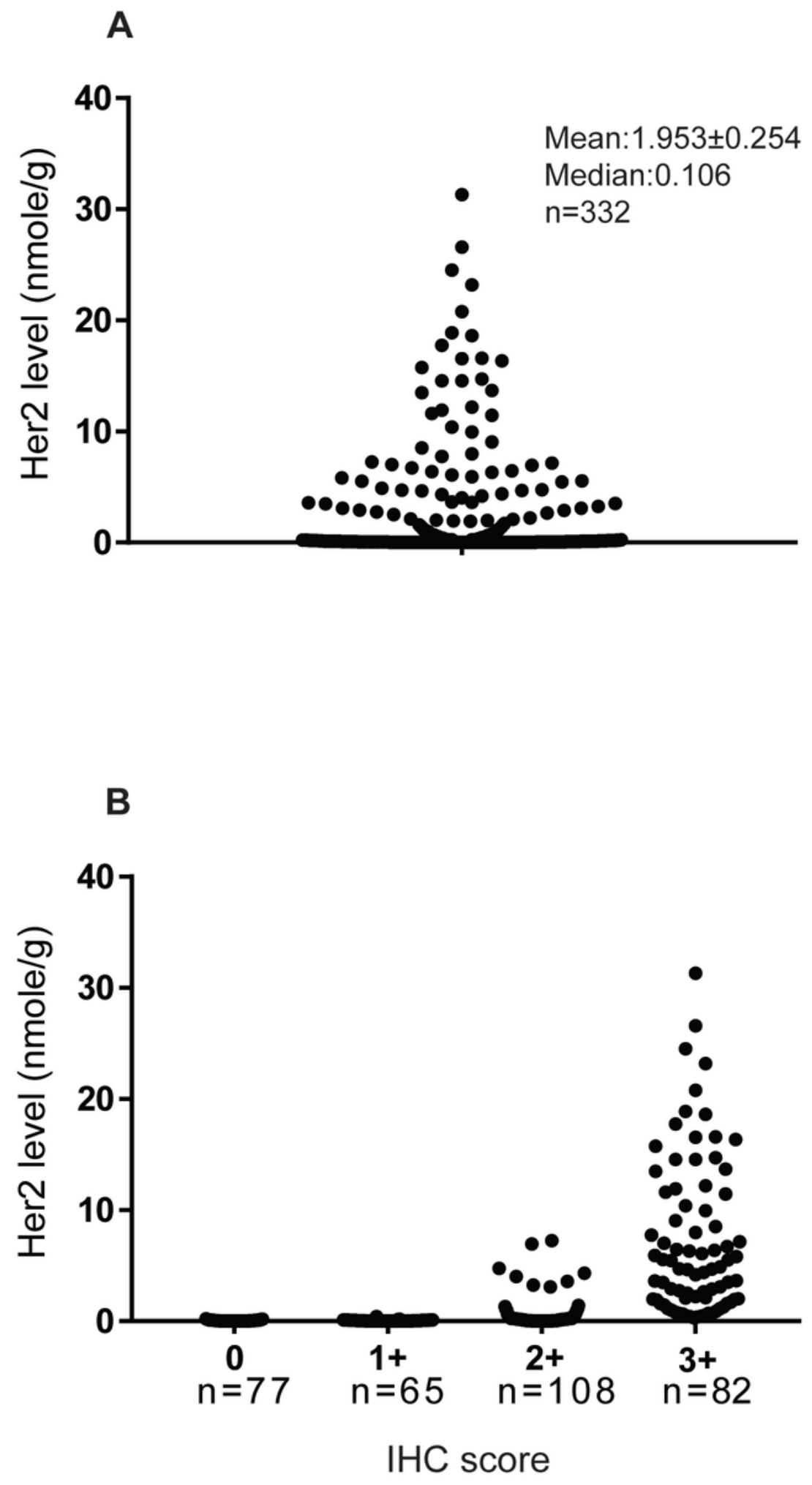

Figure 3

Distribution of all 332 samples. Her2 levels in all 332 breast cancer FFPE sample lysates were measured with QDB method using EP3 antibody. The lysates were diluted to about $0.25 \mu \mathrm{g} / \mu \mathrm{l}$, and then $2 \mu \mathrm{l}$ lysate was used for each sample. (A) the distribution of Her2 levels among 332 samples. Her2 levels were ranging from 0 (chemiluminescence readings less than two times the background) to $31.31 \mathrm{nmole} / \mathrm{g}$, with the mean at $1.953 \pm 0.254 \mathrm{nmole} / \mathrm{g}$. The 25 th percentile was at $0 \mathrm{nmole} / \mathrm{g}$ and the 75 th percentile 
was at $0.987 \mathrm{nmole} / \mathrm{g}$. (B) All samples were grouped by their IHC scores provided by local hospital. The distributions of Her2 levels in each IHC group were recorded as following: 0, 0 0.205 nmole $/ \mathrm{g}$, mean $=0.045 \pm 0.006 \mathrm{nmole} / \mathrm{g}, \mathrm{n}=77 ; 1+, 0 \sim 0.41 \mathrm{nmole} / \mathrm{g}$, mean $=0.049 \pm 0.008 \mathrm{nmole} / \mathrm{g}, \mathrm{n}=65 ; 2+, 0 \sim 7.25$ $\mathrm{nmole} / \mathrm{g}$, mean $=0.537 \pm 0.122 \mathrm{nmole} / \mathrm{g}, \mathrm{n}=108$; and $3+, 0.329 \sim 31.31 \mathrm{nmole} / \mathrm{g}$, mean $=7.12 \pm 0.773$ nmole/ $\mathrm{g}, \mathrm{n}=82$. The intra- and inter-CV were $8.98 \%$ and $9.89 \%$ respectively.

A

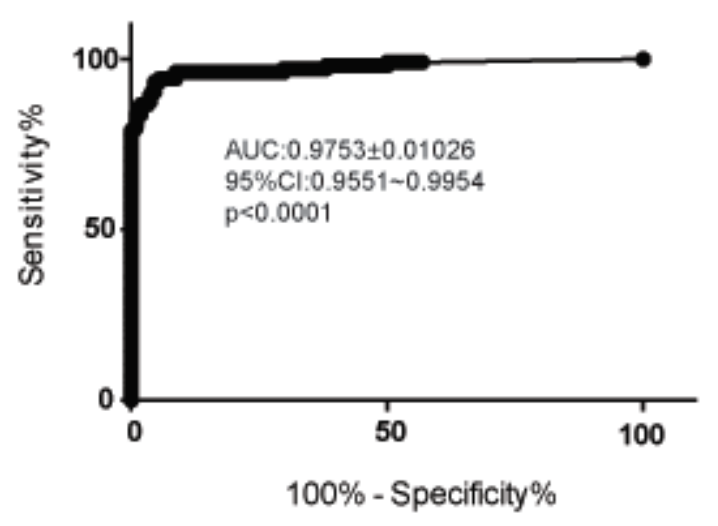

C

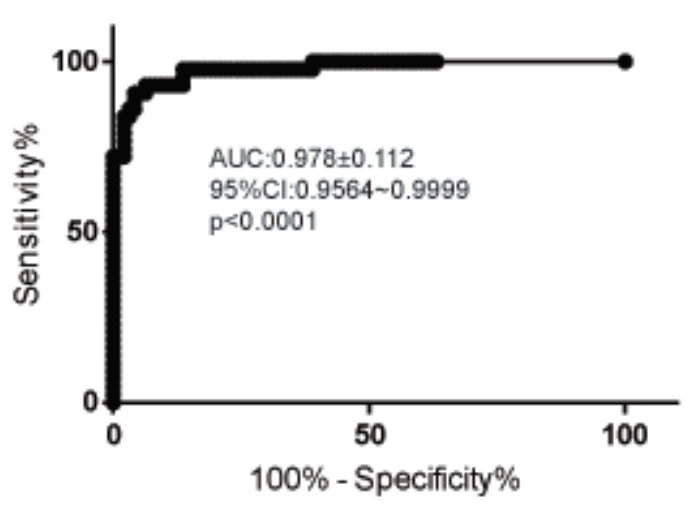

B

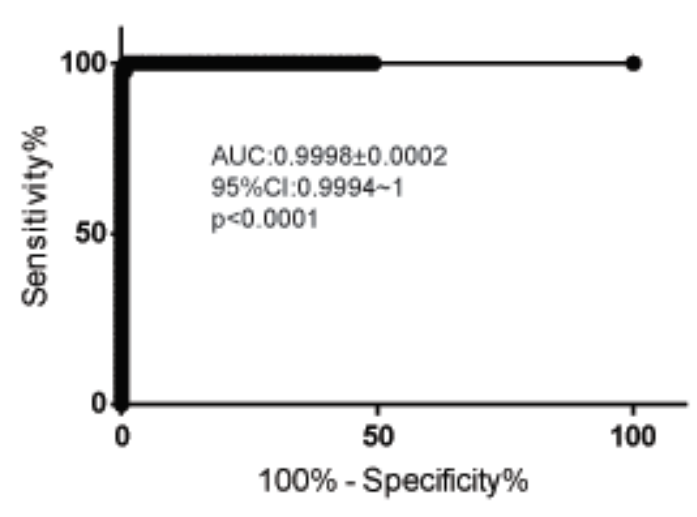

D

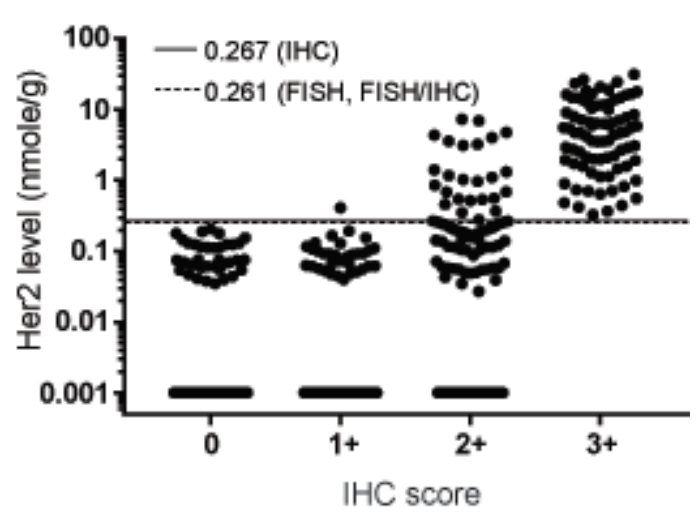

\section{Figure 4}

Evaluation of QDB results using ROC analysis with provided IHC and FISH results, Samples were separated into negative (her2-) and positive (Her2+) groups using provided results from local hospital based on the recommendations from ASCO/CAP. (A)The negative group consisted of 142 samples of IHC 0 and $1+$ and 76 samples of $\mathrm{IHC} 2+$ with FISH negative. The positive group consisted of 82 samples of $\mathrm{IHC} 3+$ and 22 samples of IHC 2+ with FISH positive. Samples scored as IHC 2+ without FISH results $(n=7)$ and with FISH results equivocal $(n=3)$ were excluded. Absolute Her2 levels from QDB analysis were used for ROC analysis with Graphpad Prism7.0 software. The ROC curve of QDB analysis was obtained with area under the Curve (AUC) at 0.9753 $\pm 0.01026 ; 95 \% \mathrm{Cl}$ : 0.9551 0.9954; $\mathrm{P}<0.0001$. (B) Samples were grouped based on their IHC scores alone, with 142 samples in the negative group (IHC 0 and 1+), and 82 samples in the positive group $(\mathrm{IHC} 3+)$. Samples with $\mathrm{IHC}$ score of $2+$ were excluded from the analysis. The ROC curve of QDB analysis was obtained with area under the Curve (AUC) at 0.9998 0.0002; 95\% Cl: 0.9994 1; P<0.0001. (C) Samples were grouped based on FISH results alone, with 95 
samples as negative (her2-) group and 43 samples as positive (Her2+) group. Equivocal cases $(n=6)$ were excluded in the analysis. The area under the curve (AUC) was at $0.978 \pm 0.0112$, with $95 \% \mathrm{Cl}$ at 0.9561 0.9999; $P<0.0001$. (D) Samples were grouped by $I H C$ scores, and the suggested cutoff values from ROC analyses in (B) at $0.267 \mathrm{nmole} / \mathrm{g}$ (solid line), in (A) (C) at $0.261 \mathrm{nmole} / \mathrm{g}$ (dashed line) were shown to demonstrate the effectiveness of these cutoff values to separate samples from Her2+ to Her2groups. Her2 levels were plotted in log scale to better demonstrate the distribution of QDB results among these samples. For those samples with undetectable Her2 level, a value of $0.001 \mathrm{nmole} / \mathrm{g}$ was arbitrarily entered to avoid omitting any sample in the log scale graph.

\section{$p<0.05$}

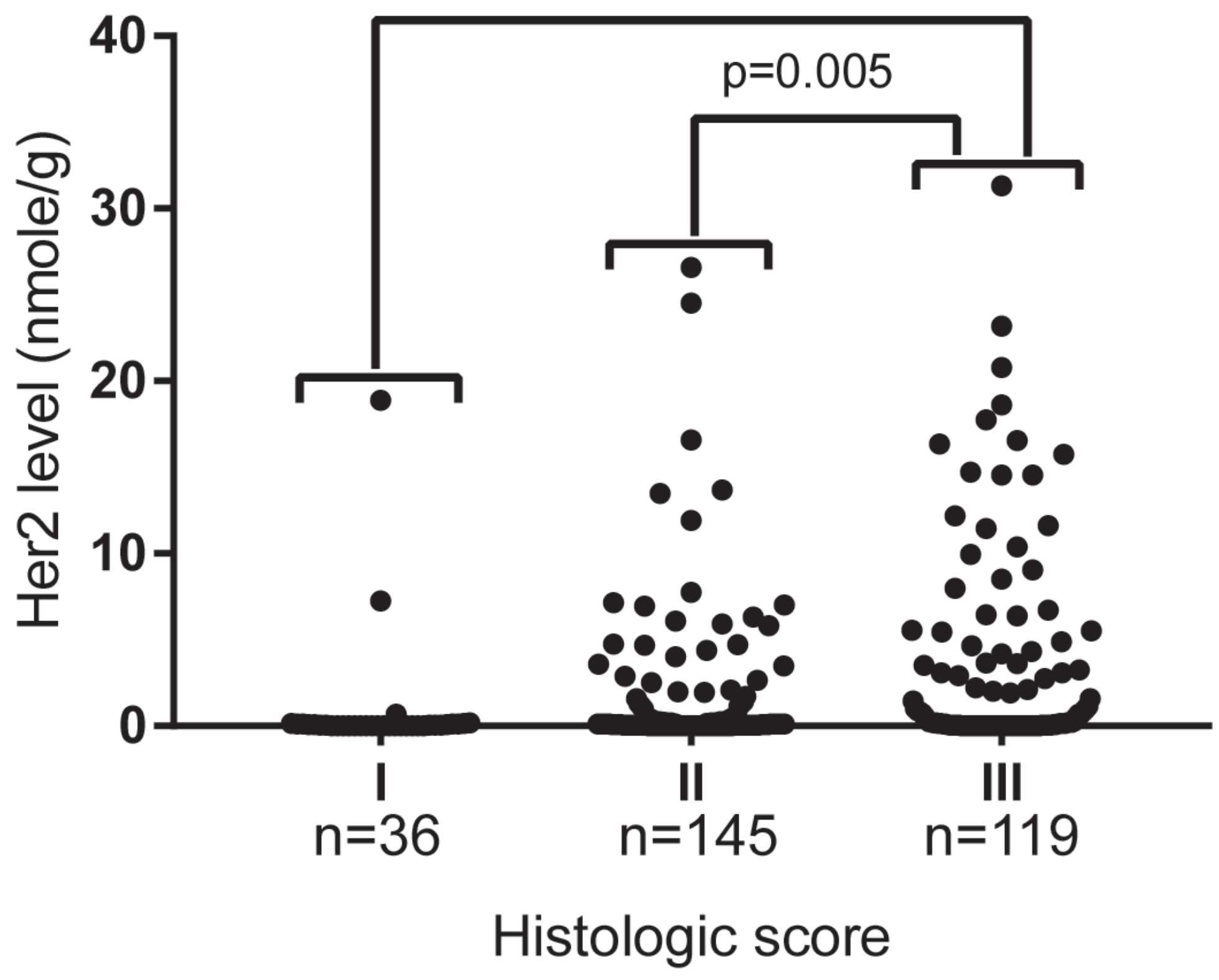

Figure 5

Assessing Her2 levels by histologic grade. The FFPE specimens (300 out of 332) were grouped according to their Nottingham histologic scores into grades I, II, and III. The Her2 levels of each grade were used for column statistics analysis with Graphpad Prism7.0 software. The mean \pm SD of the Her2 levels were 
$0.791 \pm 0.555 \mathrm{nmole} / \mathrm{g}$ for grade I $(\mathrm{n}=36), 1.554 \pm 0.330 \mathrm{nmole} / \mathrm{g}$ for grade II $(\mathrm{n}=145)$, and $3.271 \pm 0.535$ $\mathrm{nmole} / \mathrm{g}$ for grade III $(\mathrm{n}=119)$. The statistical difference was assessed with an unpaired two-tailed Student's t-test, with $p<0.05$ between grades I and III, and $p=0.005$ between grades II and III. There was no statistical difference between grade I and grade II samples.

\section{Supplementary Files}

This is a list of supplementary files associated with this preprint. Click to download.

- Additionalfile.4.pdf

- Additionalfile.3.eps

- Additionalfile.2.pdf

- Additionalfile.1.eps 